\title{
Assessment of potential toxicological aspects of dietary exposure to silicon-rich spirulina in rats
}

\author{
Joris Vidé ${ }^{a}$, Cindy Romain a ${ }^{\text {a }}$ Christine Feillet-Coudray ${ }^{\mathrm{b}}$, Béatrice Bonafos ${ }^{\mathrm{b}}$, \\ Jean Paul Cristol ${ }^{\mathrm{a}}$, Gilles Fouret ${ }^{\mathrm{b}}$, Jean-Max Rouanet ${ }^{\mathrm{a}, 1}$, Sylvie Gaillet ${ }^{\mathrm{a}, \mathrm{b}, 1, *}$ \\ a Nutrition E' Metabolism, UMR 204 NUTRIPASS, Prevention of Malnutritions E' Linked Pathologies, University of Montpellier, Place Eugène Bataillon, \\ Montpellier 34095, France \\ b UMR 866 - Dynamic Muscle E Metabolism, INRA Montpellier Center, 2 Place Viala, Montpellier 34060, France
}

\section{Keywords:}

Toxicity

Silicon

Spirulina

Rats

Oxidative status

Inflammation

\begin{abstract}
A B S T R A C T
Silicon has beneficial effects especially on bones and skin and is important in cardiovascular pathophysiology. Furthermore, in spontaneously hypertensive rats, it reduces hypertension and increases antihypertensive and antiatherogenic gene expressions in the aorta. Thus, incorporating silicon into spirulina could be a way to produce a bioavailable food supplement.

The potential toxic effects of silicon-rich spirulina (SES) through haematological and biochemical parameters and inflammatory and oxidative status were evaluated in rats' blood and liver tissue. The study consisted in a 90-day experiment on female and male rats supplemented with three doses $(28.5,57$ and $285 \mathrm{mg} / \mathrm{kg} \mathrm{BW} /$ day) of SES. No mortality, abnormal clinical signs, behavioural changes or macroscopic findings were observed whatever the groups. Haematological parameters were not modified in SES treatedgroups. No marked change was recorded in biochemical parameters The liver endogenous antioxidant enzymes (SOD, GPx, catalase) activities were not modified whatever the gender and the dose, just as markers of oxidative stress $\left(\mathrm{O}_{2}{ }^{\circ}-\right.$, TBARS, thiols $)$ and inflammation such as IL-6 and TNF-alpha.

Our findings indicate that dietary supplementation of silicon-rich spirulina on rats has no harmful side nor toxic effects and could be beneficial especially in the case of suspicion or installation of pathologies due to oxidative stress.
\end{abstract}

\section{Introduction}

Silicon is a very important element in the living world (Exley, 1998). It is soluble essentially in the form of orthosilicic acid $\mathrm{Si}(\mathrm{OH})_{4}$ in the surface waters. But beyond $2 \mathrm{mM}$ and at neutral $\mathrm{pH}$, this acid polymerizes into various forms of silica (colloidal solid) (Refitt et al., 2003) little or no soluble. At biological $\mathrm{pH}$, silicon chemistry in solution mainly relates orthosilicic acid (Knight and Kinrade, 2001) which can form stable complexes with molecules containing hydroxyl groups (Ingri, 1978). Silicon has been identified associated with various biomolecules including proteins and carbon hydrates (Bond and McAuliffe, 2003). Silicon is indispensable for cell growth and trophic tissues in humans as in animals, and as such it is an essential element. Its digestive absorption is a function of its chemical presentation. Naturally, silicon is

\footnotetext{
* Corresponding author. UMR NutriPass, CC 023, Université de Montpellier, Place Eugène Bataillon, 34095 Montpellier Cedex 05, France. Tel.: +33 467143 520; fax: +330467143521.

E-mail address: gaillet@univ-montp2.fr (S. Gaillet).

1 These authors contributed equally to this work.
}

essentially in insoluble form, so poorly available (mineral silicon). On the other hand, even in a soluble form, silicon is slightly stable because it tends to polymerize rapidly at high concentration, which is the case of orthosilicic acid $\mathrm{Si}(\mathrm{OH})_{4}$ which is the more soluble form of silicon and which has the better bioavailability (Calomme and Vanden Berghe, 1997; Jugdaohsingh et al., 2002), although it remains in a mineral form.

The silicon absorption in humans is largely performed in the form of orthosilicic acid $\mathrm{Si}(\mathrm{OH})_{4}$ found in the drinking water or formed after food hydrolysis through the digestive tract (Refitt et al., 1999). The bulk of the silicon being in the form of colloidal silica is poorly absorbed by the body. In humans, daily intake is about $20-50 \mathrm{mg}$ and the body needs 5-6 mg/day (Anonymous, 2004).

Biological importance of silicon has to be considered in the context of its body distribution. The highest concentrations are found in bone and connective tissues such as aorta, trachea, tendons and skin, where silicon appears as an actor in turnover processes through collagen and elastin synthesis (Seaborn and Nielsen, 1993). Silicon supplementation has been reported to have beneficial effects on these tissues and especially bones (Jugdaohsingh, 2007) and skin (Calomme and Vanden Berghe, 1997). Silicon deficiency, in contrast, has been associated to detrimental effects on bone mineralization and growth or skin elasticity and healing (Carlisle, 
1976). The importance of silicon has also been demonstrated in cardiovascular pathophysiology and especially in the prevention of atherosclerosis (Loeper et al., 1979). An inverse relationship between the ingestion of silicon and the development of atherosclerosis has been reported (Loeper et al., 1988), and in spontaneously hypertensive rats, silicon supplementation reduces hypertension and increases antihypertensive and antiatherogenic gene expressions in the aorta (Maehira et al., 2011). Thus, a supplementation may be justified in preventing these effects, especially in the case of suspicion or installation of pathologies.

A fundamental step is to transform silicon in an organic form that is much more assimilated by the body than mineral forms. This is what we have achieved by incorporating it into spirulina.

The cyanobacteria Spirulina platensis is commercially available for human consumption. Spirulina are a good source of vitamins and minerals and are used as nutraceutical food supplements with no toxic side effects (Chamorro et al., 1988). They are particularly suitable for the production of specific bioactive compounds that they are able to accumulate in an organically bio-transformed form. Thus, new kind of food supplements has been developed and could serve as a rich source of trace elements (Mazo et al., 2007). Silicon is one of those trace elements essential in human nutrition.

Incorporating silicon into spirulina is a way to produce a bioavailable food supplement. Thus, given the beneficial effects of silicon and the antioxidant, hypolipidaemic and anti-inflammatory properties of spirulina (Deng and Chow, 2010) a food supplement was prepared. It has been shown that spirulina had no toxic effects in vivo and in vitro and did not release toxins (Heussner et al., 2012; Yang et al., 2011).

Whereas Buesen et al. (2014) reported that silicon dioxide nanomaterials neither cause local nor systemic effects upon subacute oral administration in rats and Horie et al. (2014) demonstrated that silicon dioxide nanoparticles do not exert potent cytotoxic effects on cells in culture, others have shown that (i) silicon dioxide increased enzymatic activities in the plasma, indicating an endothelial lung damage (Deb et al., 2012), (ii) silica nanoparticles exposure triggered a pro-inflammatory status in human bronchial epithelial BEAS2B cells (Skuland et al., 2014), (iii) silicon in the form of silicon carbide induced no cytotoxicity but triggered pro-oxidative and proinflammatory responses of variable intensity on RAW 264.7 macrophages (Boudard et al., 2014).

Thus, we aimed to investigate here for the first time the safety and potential toxicity of repeated doses administration of siliconrich spirulina (for 90 days) on rats. The maximum dose administered corresponds to 5 times the daily human recommended dose by the European Food Safety Authority.

\section{Materials and methods}

\subsection{Materials}

Spirulina (produced at PhycoBiotech, Lunel, France) grown in $25 \mathrm{~m}^{3}$ pools under greenhouse with natural light, and temperature is controlled at $33^{\circ} \mathrm{C}$, in a Zarouk's medium in the presence of sodium metasilicate $\left(\mathrm{Na}_{2} \mathrm{O}_{3} \mathrm{Si}\right)$. This medium contained $\mathrm{NaHCO}_{3}, 16.8 \mathrm{~g} / \mathrm{L} ; \mathrm{K}_{2} \mathrm{HPO}_{4}, 0.5 \mathrm{~g} / \mathrm{L} ; \mathrm{NaNO}_{3}, 2.5 \mathrm{~g} / \mathrm{L} ; \mathrm{K}_{2} \mathrm{SO}_{4}, 1.0 \mathrm{~g} / \mathrm{L} ; \mathrm{NaCl}, 1.0 \mathrm{~g} / \mathrm{L} ;$ $\mathrm{MgSO}_{4} .7 \mathrm{H}_{2} 0,0.2 \mathrm{~g} / \mathrm{L} ; \mathrm{CaCl}_{3}, 0.04 \mathrm{~g} / \mathrm{L} ; \mathrm{FeSO}_{4} .7 \mathrm{H}_{2} 0,0.01 \mathrm{~g} / \mathrm{L} ; \mathrm{EDTA}, 0.08 \mathrm{~g} / \mathrm{L} ; \mathrm{H}_{3} \mathrm{BO}_{3}$, $2.86 \mathrm{mg} / \mathrm{L} ; \mathrm{MnCl}_{2} .4 \mathrm{H}_{2} \mathrm{O}, 220 \mathrm{mg} / \mathrm{L} ; \mathrm{CuSO}_{4} .5 \mathrm{H}_{2} \mathrm{O}, 79 \mathrm{mg} / \mathrm{L} ; \mathrm{MoO}_{3}, 15 \mathrm{mg} / \mathrm{L}$; and $\mathrm{Na}_{2} \mathrm{MoO}_{4}, 21 \mathrm{mg} / \mathrm{L}$ and was supplied with light aeration $(30 \mathrm{~L} / \mathrm{min})$ and the addition of $0.03 \% \mathrm{CO}_{2}$. The pool is continuously stirred with pumps. At the end of the growth, the biomass was recovered and filtered trough a $40 \mathrm{~mm}$ membrane, thoroughly washed with distilled water, frozen and lyophilized. Resultant spirulina contained $1 \%$ silicon, as indicated by the manufacturer.

\subsection{Animals and experimental design}

Thirty male and thirty female Sprague-Dawley rats (Janvier-Labs, Le Genest-StIsle, France) weighing $\sim 100 \mathrm{~g}$ were housed at $23 \pm 1{ }^{\circ} \mathrm{C}$ and were randomly divided in five groups of six animals for each sex, then subjected to a $12 \mathrm{~h}$ light/dark cycle with free access to both food and water. They were handled in compliance with European Union rules and according to the guidelines of the NIH (National Research
Council, 1985) and the Committee for Animal Care at the University of Montpellier (France).

They were fed ad libitum on a standard diet supplied by SAFE (Scientific Animal Food and Engineering, Augy, France) consisting of $236 \mathrm{~g} / \mathrm{kg}$ casein, $3.5 \mathrm{~g} / \mathrm{kg}$ L-methionine, $300 \mathrm{~g} / \mathrm{kg}$ corn starch, $30 \mathrm{~g} / \mathrm{kg}$ maltodextrin $10,290.5 \mathrm{~g} / \mathrm{kg}$ sucrose, $50 \mathrm{~g} / \mathrm{kg}$ cellulose, $45 \mathrm{~g} / \mathrm{kg}$ vegetable oil (10.3\% energy of the diet), $35 \mathrm{~g} / \mathrm{kg}$ mineral mix and $10 \mathrm{mg} / \mathrm{kg}$ vitamin mix. Vitamin and mineral mixes were formulated according to AIN-93 guidelines (Reeves et al., 1993). Rats and uneaten food were weighed every day. For each sex, rats of each group received daily by gavage either tap water (Control, CTR) or an aqueous suspension of crude spirulina (SP) at a dose of $285 \mathrm{mg} / \mathrm{kg} \mathrm{BW}$, or silicon-rich spirulina (SES) at a dose of $28.5 \mathrm{mg} / \mathrm{kg}$ BW (SES1 group) or SES at a dose of $57 \mathrm{mg} / \mathrm{kg}$ BW (SES2 group), or SES at a dose of $285 \mathrm{mg} / \mathrm{kg}$ BW (SES3) for 90 days. All animals were observed twice daily for general appearance, behaviour, signs of morbidity and mortality (once before treatment and once after). The SES2 dose ( $57 \mathrm{mg} / \mathrm{kg}$ BW/day) corresponds to the EFSA recommendations adapted to the rat (EFSA, 2004). The SES1 dose $(28.5 \mathrm{mg} / \mathrm{kg} \mathrm{BW} / \mathrm{d})$ is a two-fold lower dose, while the SES3 dose is fivefold times higher than SES2.

\subsection{Sampling}

At the end of the experimental period, rats were deprived of food overnight and blood samples were collected under anaesthesia by cardiac puncture. The liver was perfused with $0.15 \mathrm{M} \mathrm{NaCl}$ to remove residual blood, rapidly excised, weighed, sectioned for analyses, and stored at $-80^{\circ} \mathrm{C}$. Heart, spleen and kidneys were rapidly excised, blotted dry and weighed.

\subsection{Haematological and biochemical plasma parameters}

All samples were collected early in the working day to reduce biological variation. Test tubes containing EDTA, as an anticoagulant, were used for haematology. Haematological parameters (red blood cells (RBCs), haemoglobin, haematocrit, mean corpuscular volume (MCV), mean corpuscular haemoglobin $(\mathrm{MCH})$, mean corpuscular haemoglobin concentration (MCHC), white blood cells (WBCs), neutrophils, eosinophils, basophils, lymphocytes, monocytes, platelets) were determined with an ABX Pentra DX 120 whole blood automated analyser (Horiba ABX, Montpellier, France).

Heparinized tubes were used for blood biochemistry. All parameters (urea, creatinine, cholesterol $(\mathrm{CH})$, triglyceride $(\mathrm{TG})$, chloride $(\mathrm{Cl})$, calcium $(\mathrm{Ca})$, phosphorus (P), bilirubin, aspartate transaminase (ASAT), alanine transaminase (ALAT) and gammaglutamyl transferase (GGT)) were determined in plasma samples, after centrifugation (2500 rpm, $15 \mathrm{~min}$ ), with a Cobas ${ }^{\circledR} 6000$ automated analyser (Roche, Meylan, France).

\subsection{Oxidative status}

Liver superoxide anion $\left(\mathrm{O}_{2}{ }^{\circ-}\right)$ production by NADPH oxidase were evaluated by the intensity of lucigenin-enhanced chemiluminescence ( $10 \mu \mathrm{M}$ lucigenin), measured with a luminometer (Perkin Elmer Wallac, Victor, Turku, Finland) on tissue homogenates, as previously described (Romain et al., 2012). Results were expressed as relative light units (RLU/mg protein).

The index of lipid peroxidation was determined from the liver homogenate by measuring thiobarbituric acid reactive species (TBARS) according to Sunderman et al. (1985). After thiobarbituric acid had been reacted with malondialdehyde, the reaction product was measured spectrometrically at $532 \mathrm{~nm}$. Optical imperfections were corrected according to Allen (1950) at 508 and $556 \mathrm{~nm}$. Results were expressed as nanomoles of TBARS per gram of tissue.

Total thiols (SH) were determined according to Faure and Lafond (1995). The method is based on the reaction of 5,5'-dithiobis (2-nitrobenzoic) (DTNB) with the samples producing a yellow product, the thionitrobenzoic acid (TNB), measured spectrometrically at $412 \mathrm{~nm}$. Results were expressed as nanomoles of SH per milligram of protein.

Protein content was determined by using a commercial protein assay (Sigma, Saint Quentin Fallavier, France) according to the method of Smith et al. (1985) and using bovine serum albumin as standard.

Liver tissue was homogenized in $50 \mathrm{mM}$ phosphate buffer ( $\mathrm{pH} 7.0$ ) and spun at $3500 \mathrm{~g}$ for $15 \mathrm{~min}$ at $4{ }^{\circ} \mathrm{C}$. The superoxide dismutase activity (SOD) was assayed in the supernatant using the method of Marklund and Marklund (1974) and Marklund (1976), based on a competition between the oxidation reaction of pyrogallol by $\mathrm{O}_{2}{ }^{\circ}$ and the dismutation of $\mathrm{O}_{2}{ }^{\circ-}$ by SOD. The reaction product was measured spectrometrically at $320 \mathrm{~nm}$. Results were expressed as unity activity per milligram of protein. The glutathione peroxidase activity (GPx) was evaluated using the method of Flohé and Günzler (1984), and activity was expressed as milli-units per milligram of protein. The activity of catalase (CAT) was measured according to Beers and Sizer (1952). The enzyme catalyses the decomposition of hydrogen peroxide into water molecule. Activity was expressed as units per milligram of protein.

\subsection{Inflammatory factors evaluation}

For pro-inflammatory cytokines determination, liver tissue was homogenized in $10 \mathrm{mM}$ Tris buffer ( $\mathrm{pH} 7.4$ ) containing $2 \mathrm{M} \mathrm{NaCl}, 1 \mathrm{mM}$ EDTA, $0.01 \%$ Tween $80,1 \mathrm{mM}$ phenylmethylsulfonyl fluoride, and centrifuged at $9000 \mathrm{~g}$ for $30 \mathrm{~min}$ at $4{ }^{\circ} \mathrm{C}$. The 
resultant supernatant was used for cytokines determination. Liver tumour-necrosisfactor-alpha (TNF day $\alpha$ ) and interleukin- 6 (IL-6) levels were quantified by ELISA using commercial kits from R\&D Systems (Lille, France).

\subsection{Statistical analyses}

Data are shown as means \pm SEM. Statistical analysis of the data was carried out using StatView IV software (Abacus Concept, Berkeley, CA) by one-way ANOVA followed by Student's t-test. Differences were considered significant at $\mathrm{p}<\mathbf{0 . 0 5}$.

\section{Results and discussion}

\subsection{Repeated dose (90 days) toxicological evaluation}

All animals survived the 90-day treatment period. No abnormal clinical signs, behavioural changes or macroscopic findings were observed. No significant differences in food (Table 1) and water consumption were noted among groups. As shown in Table 1, there are no significant differences in body weight gain. As far as the major organs' weight, there was no effect of the treatment, except in female heart weight SES2 which was however to the limit of significance $(\mathrm{p}=0.0476)$.

\subsection{Haematological and biochemical parameters}

Some haematological parameters differed between control and some treated groups (Table 2). In female rats, a 10\% decrease in red blood cells number appeared just as a significant basophils per cent increase in SES2 group compared to CTR group. There are no differences in male groups. Moreover, all values were within usual limits (Bailly and Duprat, 1990; Fallon, 1996).

As shown in Table 3, biochemical parameters were not affected whatever the group except in female rats, who showed $29 \%$ $(\mathrm{p}=0.0028), 21 \%(\mathrm{p}=0.0223)$ and $38 \%(\mathrm{p}=0.0002)$ reduced ALAT activity in SP, SES2 and SES3 groups respectively compared to controls. In male rats, no parameter changed regardless of the dose supplied. Female rats also exhibited reduced triglyceride levels whereas males did not. However, the data were within normal ranges according to Moraillon et al. (2010).

\subsection{Liver oxidative status}

$\mathrm{O}_{2}{ }^{\circ-}$ is the main free radical and we measured its production in the liver via the NADPH oxidase enzyme activity. TBARS level was analysed as a marker of lipid peroxidation, and total thiols level was measured to evaluate the extent of oxidative damage proteins. For these three parameters of the hepatic oxidative stress, there is no difference between the groups, with the exception of the female group SES3 (Table 4). Only TBARS level for SES3 female group was increased by $97.5 \%$ compared to CTR group and it is likely due to two animals in the group that responded differently, leading to high average and SEM values. However, the activity of NADPH oxidase, a pro-oxidative enzyme, measured through the $\mathrm{O}_{2}{ }^{\circ-}$ production, just as thiols content were not modified regardless of the SES dose. These findings suggest that SES treatment did not induce any oxidative stress. Moreover, females showed in particular a smaller $\mathrm{O}_{2}{ }^{\circ-}$ production and a lower thiols rate than males, that could be explained by an oestrogenic action since it is known to protect against oxidation (Chakrabarti et al., 2014; Shwaery et al., 1998).

Liver antioxidant enzyme activities SOD, GPx and CAT were not modified by SES treatment (Table 5 ). As seen above for $\mathrm{O}_{2}{ }^{{ }^{-}}$production and thiols, higher SOD and GPx activities appeared in females due to oestrogenic hormones (Borrás et al., 2010; Wu et al., 2014).

\subsection{Liver inflammatory status}

The IL- 6 and TNF- $\alpha$ levels were measured as pro-inflammatory markers. As shown in Table 6, both levels were not significantly different among groups. This suggests that SES treatment does not trigger an inflammatory status, which reinforces the values obtained on oxidative stress status. In fact, oxidative stress and inflammation are closely linked. The pathways that generate mediators of inflammation such as interleukins are closely linked to oxidative stress (Reuter et al., 2010).

\section{Conclusions}

This study represents the first standard toxicological data on silicon-rich spirulina. No haematological nor biochemical anomalies were noted. SES treatment did not reveal any changes in food

Table 1

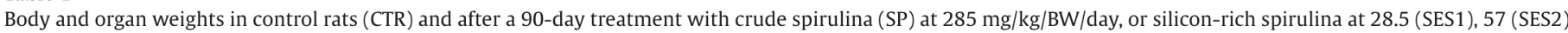
and $285 \mathrm{mg} / \mathrm{kg}$ BW/day (SES3). ${ }^{\mathrm{a}}$

\begin{tabular}{|c|c|c|c|c|c|}
\hline & \multicolumn{5}{|l|}{ Female } \\
\hline & CTR & SP & SES1 & SES2 & SES3 \\
\hline Food intake (g/day) & $21.3 \pm 2.2$ & $19.6 \pm 3.6$ & $21.6 \pm 2.8$ & $21.0 \pm 1.9$ & $21.9 \pm 2.3$ \\
\hline Body weight gain (g) & $216 \pm 17$ & $184 \pm 8$ & $193 \pm 6$ & $186 \pm 10$ & $213 \pm 12$ \\
\hline \multicolumn{6}{|l|}{ Organ weight (\% BW) } \\
\hline Liver & $3.00 \pm 0.14$ & $3.27 \pm 0.12$ & $3.03 \pm 0.14$ & $3.09 \pm 0.08$ & $2.85 \pm 0.20$ \\
\hline Heart & $0.34 \pm 0.01$ & $0.37 \pm 0.01$ & $0.37 \pm 0.02$ & $0.38 \pm 0.01^{*}$ & $0.35 \pm 0.01$ \\
\hline Rate & $0.17 \pm 0.01$ & $0.20 \pm 0.15$ & $0.19 \pm 0.01$ & $0.19 \pm 0.01$ & $0.19 \pm 0.01$ \\
\hline \multirow[t]{3}{*}{ Kidneys } & $0.61 \pm 0.01$ & $0.67 \pm 0.17$ & $0.67 \pm 0.03$ & $0.66 \pm 0.01$ & $0.67 \pm 0.03$ \\
\hline & \multicolumn{5}{|l|}{ Male } \\
\hline & CTR & SP & SES1 & SES2 & SES3 \\
\hline Food intake (g/day) & $30.8 \pm 3.9$ & $29.7 \pm 3.2$ & $30.8 \pm 3.0$ & $29.9 \pm 4.0$ & $30.3 \pm 3.7$ \\
\hline Body weight gain (g) & $469 \pm 19$ & $476 \pm 37$ & $513 \pm 23$ & $476 \pm 24$ & $458 \pm 18$ \\
\hline \multicolumn{6}{|l|}{ Organ weight (\% BW) } \\
\hline Liver & $2.50 \pm 0.13$ & $2.72 \pm 0.13$ & $2.65 \pm 0.06$ & $2.50 \pm 0.13$ & $2.55 \pm 0.12$ \\
\hline Heart & $0.28 \pm 0.01$ & $0.32 \pm 0.03$ & $0.28 \pm 0.01$ & $0.29 \pm 0.01$ & $0.29 \pm 0.01$ \\
\hline Rate & $0.23 \pm 0.01$ & $0.26 \pm 0.02$ & $0.21 \pm 0.01$ & $0.24 \pm 0.01$ & $0.25 \pm 0.01$ \\
\hline Kidneys & $0.52 \pm 0.01$ & $0.53 \pm 0.01$ & $0.51 \pm 0.02$ & $0.54 \pm 0.01$ & $0.55 \pm 0.02$ \\
\hline
\end{tabular}

a Data are expressed as mean + SEM.

* $\mathrm{P}<0.05$ compared to untreated rats (CTR group). 
Table 2

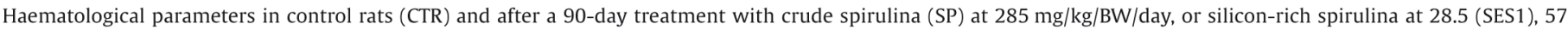
(SES2) and $285 \mathrm{mg} / \mathrm{kg} \mathrm{BW} /$ day (SES3). ${ }^{a}$

\begin{tabular}{|c|c|c|c|c|c|}
\hline & \multicolumn{5}{|l|}{ Female } \\
\hline & CTR & SP & SES1 & SES2 & SES3 \\
\hline $\mathrm{RBC}\left(\times 10^{4} / \mathrm{mm}^{3}\right)$ & $720 \pm 17$ & $707 \pm 16$ & $729 \pm 26$ & $647 \pm 38^{*}$ & $703 \pm 28$ \\
\hline Haemoglobin $(\mathrm{g} / \mathrm{dL})$ & $14.40 \pm 0.40$ & $14.18 \pm 0.48$ & $13.38 \pm 0.48$ & $13.40 \pm 0.20$ & $14.27 \pm 0.59$ \\
\hline Haematocrit (\%) & $38.90 \pm 0.93$ & $39.70 \pm 1.25$ & $39.80 \pm 1.53$ & $37.30 \pm 0.40$ & $39.40 \pm 1.25$ \\
\hline $\operatorname{MCV}(\mathrm{fl})$ & $53.80 \pm 0.80$ & $54.30 \pm 0.76$ & $54.50 \pm 0.56$ & $54.30 \pm 0.33$ & $54.20 \pm 0.75$ \\
\hline $\mathrm{MCH}(\mathrm{pg})$ & $20.0 \pm 0.40$ & $20.10 \pm 0.29$ & $19.00 \pm 0.50$ & $19.60 \pm 0.31$ & $20.30 \pm 0.30$ \\
\hline $\operatorname{MCHC}(\mathrm{g} / \mathrm{dL})$ & $36.90 \pm 0.28$ & $35.80 \pm 0.63$ & $35.90 \pm 0.97$ & $35.90 \pm 0.58$ & $36.20 \pm 0.40$ \\
\hline $\mathrm{WBC}\left(\times 10^{2} / \mathrm{mm}^{3}\right)$ & $27.0 \pm 4.0$ & $24.0 \pm 1.4$ & $28.0 \pm 2.7$ & $25.0 \pm 4.8$ & $28.0 \pm 3.4$ \\
\hline Neutrophils (\%) & $12.00 \pm 1.74$ & $13.50 \pm 1.15$ & $13.20 \pm 1.03$ & $12.40 \pm 0.72$ & $13.00 \pm 0.80$ \\
\hline Eosinophils (\%) & $0.60 \pm 0.18$ & $0.60 \pm 0.25$ & $0.50 \pm 0.16$ & $0.90 \pm 0.16$ & $0.70 \pm 0.24$ \\
\hline Basophils (\%) & $0.17 \pm 0.03$ & $0.23 \pm 0.03$ & $0.28 \pm 0.03$ & $0.32 \pm 0.06^{*}$ & $0.23 \pm 0.04$ \\
\hline Lymphocytes (\%) & $86.3 \pm 1.9$ & $84.4 \pm 1.1$ & $85.0 \pm 1.2$ & $85.8 \pm 0.7$ & $85.0 \pm 0.9$ \\
\hline Monocytes (\%) & $0.9 \pm 0.1$ & $1.3 \pm 0.2$ & $1.0 \pm 0.1$ & $0.6 \pm 0.0^{*}$ & $1.0 \pm 0.1$ \\
\hline \multirow[t]{3}{*}{ Platelets $\left(\times 10^{3} / \mathrm{mm}^{3}\right)$} & $697 \pm 82$ & $698 \pm 76$ & $710 \pm 63$ & $638 \pm 112$ & $597 \pm 43$ \\
\hline & \multicolumn{5}{|l|}{ Male } \\
\hline & CTR & SP & SES1 & SES2 & SES3 \\
\hline $\mathrm{RBC}\left(\times 10^{4} / \mathrm{mm}^{3}\right)$ & $821 \pm 40$ & $791 \pm 47$ & $757 \pm 77$ & $823 \pm 20$ & $732 \pm 38$ \\
\hline Haemoglobin (g/dL) & $14.82 \pm 0.63$ & $14.95 \pm 0.99$ & $13.70 \pm 1.29$ & $14.98 \pm 0.21$ & $13.86 \pm 0.72$ \\
\hline Haematocrit (\%) & $41.60 \pm 1.81$ & $42.80 \pm 2.35$ & $40.80 \pm 3.86$ & $42.20 \pm 0.66$ & $39.50 \pm 1.86$ \\
\hline $\operatorname{MCV}(\mathrm{fl})$ & $50.80 \pm 0.86$ & $51.30 \pm 0.33$ & $51.80 \pm 0.60$ & $51.50 \pm 0.86$ & $52.20 \pm 0.54$ \\
\hline $\mathrm{MCH}(\mathrm{pg})$ & $18.10 \pm 0.37$ & $18.90 \pm 0.59$ & $18.30 \pm 0.21$ & $18.30 \pm 0.28$ & $18.60 \pm 0.20$ \\
\hline $\operatorname{MCHC}(\mathrm{g} / \mathrm{dL})$ & $35.70 \pm 0.37$ & $36.70 \pm 1.01$ & $35.30 \pm 0.29$ & $35.50 \pm 0.13$ & $35.60 \pm 0.21$ \\
\hline $\operatorname{WBC}\left(\times 10^{2} / \mathrm{mm}^{3}\right)$ & $68.0 \pm 8.7$ & $66.0 \pm 1.1$ & $54.0 \pm 7.4$ & $58.0 \pm 6.6$ & $60.0 \pm 1.1$ \\
\hline Neutrophils (\%) & $10.20 \pm 1.55$ & $11.00 \pm 1.37$ & $10.20 \pm 0.61$ & $11.20 \pm 1.91$ & $7.90 \pm 0.46$ \\
\hline Eosinophils (\%) & $0.40 \pm 0.18$ & $0.60 \pm 0.16$ & $0.40 \pm 0.14$ & $0.30 \pm 0.03$ & $0.40 \pm 0.08$ \\
\hline Basophils (\%) & $0.10 \pm 0.03$ & $0.13 \pm 0.04$ & $0.20 \pm 0.09$ & $0.13 \pm 0.05$ & $0.12 \pm 0.03$ \\
\hline Lymphocytes (\%) & $87.2 \pm 1.7$ & $86.4 \pm 1.6$ & $87.5 \pm 1.0$ & $86.6 \pm 1.9$ & $98.5 \pm 0.5$ \\
\hline Monocytes (\%) & $2.0 \pm 0.4$ & $1.9 \pm 0.3$ & $1.7 \pm 0.4$ & $1.8 \pm 0.1$ & $2.1 \pm 0.6$ \\
\hline Platelets $\left(\times 10^{3} / \mathrm{mm}^{3}\right)$ & $646 \pm 71$ & $602 \pm 74$ & $528 \pm 74$ & $590 \pm 782$ & $561 \pm 97$ \\
\hline
\end{tabular}

a Data are expressed as mean + SEM

* $\mathrm{P}<0.05$ compared to untreated rats (CTR group).

Table 3

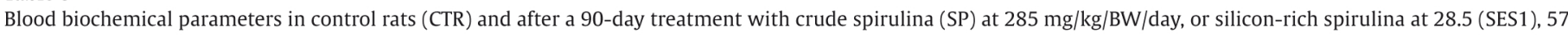
(SES2) and $285 \mathrm{mg} / \mathrm{kg} \mathrm{BW} /$ day (SES3). ${ }^{\mathrm{a}}$

\begin{tabular}{|c|c|c|c|c|c|}
\hline & \multicolumn{5}{|l|}{ Female } \\
\hline & CTR & SP & SES1 & SES2 & SES3 \\
\hline Urea $(\mathrm{mmol} / \mathrm{L})$ & $5.04 \pm 0.43$ & $4.79 \pm 0.46$ & $5.93 \pm 0.36$ & $5.51 \pm 0.47$ & $4.72 \pm 0.31$ \\
\hline Creatinine $(\mu \mathrm{mol} / \mathrm{L})$ & $32.28 \pm 2.16$ & $32.77 \pm 3.11$ & $34.82 \pm 1.31$ & $36.00 \pm 4.20$ & $30.68 \pm 1.38$ \\
\hline $\mathrm{CH}(\mathrm{mmol} / \mathrm{L})$ & $2.74 \pm 0.32$ & $2.51 \pm 0.40$ & $2.38 \pm 0.27$ & $1.97 \pm 0.08$ & $2.01 \pm 0.12$ \\
\hline $\mathrm{TG}(\mathrm{mmol} / \mathrm{L})$ & $2.21 \pm 0.53$ & $0.80 \pm 0.13^{*}$ & $0.90 \pm 0.17^{*}$ & $0.64 \pm 0.06^{*}$ & $1.14 \pm 0.11$ \\
\hline $\mathrm{Cl}(\mathrm{mmol} / \mathrm{L})$ & $97.30 \pm 0.84$ & $98.20 \pm 0.70$ & $98.50 \pm 0.85$ & $98.30 \pm 0.33$ & $98.00 \pm 0.45$ \\
\hline $\mathrm{Ca}(\mathrm{mmol} / \mathrm{L})$ & $2.72 \pm 0.04$ & $2.70 \pm 0.05$ & $2.66 \pm 0.06$ & $2.65 \pm 0.02$ & $2.70 \pm 0.03$ \\
\hline $\mathrm{P}(\mathrm{mmol} / \mathrm{L})$ & $1.71 \pm 0.10$ & $1.87 \pm 0.15$ & $1.49 \pm 0.08^{*}$ & $1.85 \pm 0.07$ & $2.00 \pm 0.15$ \\
\hline Bilirubin $(\mu \mathrm{mol} / \mathrm{L})$ & $2.50 \pm 0.22$ & $2.33 \pm 0.21$ & $2.00 \pm 0.00$ & $2.33 \pm 0.21$ & $2.33 \pm 0.21$ \\
\hline $\operatorname{ASAT}(\mathrm{U} / \mathrm{L})^{\mathrm{b}}$ & $85.60 \pm 4.21$ & $86.20 \pm 6.17$ & $82.40 \pm 7.84$ & $86.40 \pm 3.98$ & $75.83 \pm 3.14$ \\
\hline $\operatorname{ALAT}(\mathrm{U} / \mathrm{L})^{\mathrm{b}}$ & $37.2 \pm 2.35$ & $26.20 \pm 2.92^{*}$ & $31.00 \pm 2.70$ & $29.50 \pm 1.65^{*}$ & $23.00 \pm 1.63^{*}$ \\
\hline \multirow[t]{3}{*}{ GGT $(\mathrm{UI} / \mathrm{L})^{\mathrm{b}}$} & $3.33 \pm 0.66$ & $3.80 \pm 0.37$ & $4.00 \pm 0.68$ & $3.33 \pm 0.42$ & $4.50 \pm 0.50$ \\
\hline & \multicolumn{5}{|l|}{ Male } \\
\hline & CTR & SP & SES1 & SES2 & SES3 \\
\hline Urea (mmol/L) & $5.73 \pm 0.15$ & $6.04 \pm 0.31$ & $6.57 \pm 0.44$ & $6.54 \pm 0.22$ & $6.51 \pm 0.32$ \\
\hline Creatinine $(\mu \mathrm{mol} / \mathrm{L})$ & $38.80 \pm 1.03$ & $40.28 \pm 0.99$ & $39.08 \pm 1.73$ & $39.23 \pm 1.57$ & $37.63 \pm 2.78$ \\
\hline $\mathrm{CH}(\mathrm{mmol} / \mathrm{L})$ & $1.71 \pm 0.09$ & $1.57 \pm 0.03$ & $1.71 \pm 0.08$ & $1.51 \pm 0.09$ & $1.50 \pm 0.06$ \\
\hline $\mathrm{TG}(\mathrm{mmol} / \mathrm{L})$ & $1.03 \pm 0.08$ & $1.04 \pm 0.15$ & $1.31 \pm 0.17$ & $1.14 \pm 0.07$ & $0.98 \pm 0.14$ \\
\hline $\mathrm{Cl}(\mathrm{mmol} / \mathrm{L})$ & $98.50 \pm 1.15$ & $97.50 \pm 0.56$ & $97.20 \pm 0.31$ & $98.00 \pm 0.63$ & $98.00 \pm 0.84$ \\
\hline $\mathrm{Ca}(\mathrm{mmol} / \mathrm{L})$ & $2.68 \pm 0.06$ & $2.62 \pm 0.02$ & $2.68 \pm 0.02$ & $2.68 \pm 0.02$ & $2.65 \pm 0.05$ \\
\hline $\mathrm{P}(\mathrm{mmol} / \mathrm{L})$ & $2.28 \pm 0.08$ & $2.25 \pm 0.05$ & $2.41 \pm 0.72$ & $2.27 \pm 0.06$ & $2.46 \pm 0.05$ \\
\hline Bilirubin $(\mu \mathrm{mol} / \mathrm{L})$ & $2.17 \pm 0.17$ & $2.00 \pm 0.00$ & $2.00 \pm 0.00$ & $2.17 \pm 0.17$ & $2.00 \pm 0.00$ \\
\hline $\operatorname{ASAT}(U / L)^{\mathrm{b}}$ & $106.60 \pm 9.13$ & $97.00 \pm 2.70$ & $90.60 \pm 6.30$ & $93.80 \pm 5.72$ & $96.33 \pm 4.46$ \\
\hline $\operatorname{ALAT}(\mathrm{U} / \mathrm{L})^{\mathrm{b}}$ & $46.80 \pm 1.99$ & $43.80 \pm 2.76$ & $44.80 \pm 3.20$ & $41.80 \pm 3.66$ & $44.60 \pm 3.96$ \\
\hline GGT $(\mathrm{UI} / \mathrm{L})^{\mathrm{b}}$ & $0.83 \pm 0.17$ & $1.00 \pm 0.00$ & $1.33 \pm 0.33$ & $1.00 \pm 0.00$ & $0.83 \pm 0.17$ \\
\hline
\end{tabular}

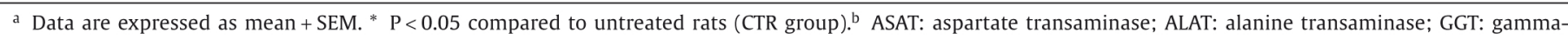
glutamyl transferase. 
Table 4

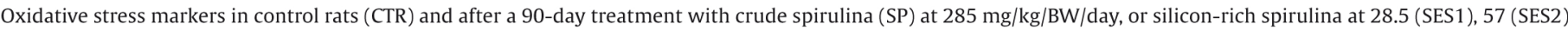
and $285 \mathrm{mg} / \mathrm{kg}$ BW/day (SES3). ${ }^{\text {a }}$

\begin{tabular}{|c|c|c|c|c|c|}
\hline & \multicolumn{5}{|l|}{ Female } \\
\hline & CTR & SP & SES1 & SES2 & SES3 \\
\hline $\mathrm{O}_{2}{ }^{\circ-}$ (RLU/mg prot) & $1526 \pm 229$ & $1581 \pm 222$ & $1292 \pm 134$ & $1407 \pm 234$ & $1241 \pm 178$ \\
\hline TBARS (nmol/g) & $292.2 \pm 65.0$ & $323.3 \pm 88.4$ & $389.9 \pm 79.9$ & $370.6 \pm 88.6$ & $577.3 \pm 162.7^{*}$ \\
\hline \multirow[t]{3}{*}{ SH (nmol/mg prot) } & $98.0 \pm 23.9$ & $77.1 \pm 24.7$ & $81.3 \pm 29.5$ & $79.4 \pm 17.0$ & $75.0 \pm 18.1$ \\
\hline & \multicolumn{5}{|l|}{ Male } \\
\hline & CTR & SP & SES1 & SES2 & SES3 \\
\hline $\mathrm{O}_{2}{ }^{\circ-}(\mathrm{RLU} / \mathrm{mg}$ prot $)$ & $2432 \pm 278$ & $2593 \pm 311$ & $2312 \pm 360$ & $2115 \pm 274$ & $1947 \pm 249$ \\
\hline TBARS (nmol/g) & $193.8 \pm 64.4$ & $191.3 \pm 32.1$ & $208.7 \pm 36.6$ & $227.9 \pm 45.0$ & $219.9 \pm 45.3$ \\
\hline $\mathrm{SH}$ (nmol/mg prot) & $87.4 \pm 27.3$ & $107.7 \pm 13.4$ & $102.2 \pm 13.4$ & $105.8 \pm 10.1$ & $101.2 \pm 10.4$ \\
\hline
\end{tabular}

a Data are expressed as mean $+\mathrm{SEM}$

* $\mathrm{P}<0.05$ compared to untreated rats (CTR group).

Table 5

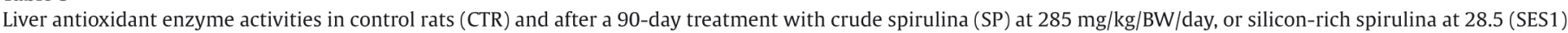
57 (SES2) and $285 \mathrm{mg} / \mathrm{kg}$ BW/day (SES3). ${ }^{\text {a }}$

\begin{tabular}{|c|c|c|c|c|c|}
\hline & \multicolumn{5}{|l|}{ Female } \\
\hline & CTR & SP & SES1 & SES2 & SES3 \\
\hline SOD (U/mg prot) & $20.3 \pm 1.8$ & $20.9 \pm 2.9$ & $20.9 \pm 3.3$ & $20.7 \pm 3.7$ & $21.6 \pm 2.5$ \\
\hline GPx (mU/mg prot) & $6865 \pm 202$ & $6674 \pm 571$ & $7091 \pm 892$ & $6694.4 \pm 384$ & $7421 \pm 636$ \\
\hline \multirow[t]{3}{*}{ CAT (U/mg prot) } & $702 \pm 89$ & $691 \pm 112$ & $676 \pm 175$ & $628 \pm 82$ & $667 \pm 98$ \\
\hline & \multicolumn{5}{|l|}{ Male } \\
\hline & CTR & SP & SES1 & SES2 & SES3 \\
\hline SOD (U/mg prot) & $17.5 \pm 1.8$ & $17.7 \pm 3.1$ & $18.2 \pm 2.1$ & $18.3 \pm 2.6$ & $17.2 \pm 1.6$ \\
\hline GPx (mU/mg prot) & $3338 \pm 562$ & $4203 \pm 886$ & $3674 \pm 496$ & $3884 \pm 420$ & $3556 \pm 533$ \\
\hline CAT (U/mg prot) & $728 \pm 96$ & $772 \pm 134$ & $711 \pm 42$ & $787 \pm 108$ & $749 \pm 119$ \\
\hline
\end{tabular}

a Data are expressed as mean + SEM.

Table 6

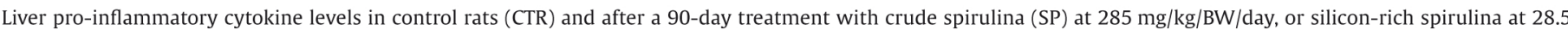
(SES1), 57 (SES2) and $285 \mathrm{mg} / \mathrm{kg}$ BW/day (SES3). ${ }^{\mathrm{a}}$

\begin{tabular}{|c|c|c|c|c|c|}
\hline & \multicolumn{5}{|l|}{ Female } \\
\hline & CTR & SP & SES1 & SES2 & SES3 \\
\hline IL-6 (pg/mg prot) & $726.8 \pm 149.9$ & $719.2 \pm 217.8$ & $611.9 \pm 209.5$ & $849.7 \pm 274.5$ & $649.41 \pm 125.1$ \\
\hline \multirow[t]{3}{*}{ TNF- $\alpha$ (pg/mg prot) } & $417.6 \pm 99.1$ & $299.0 \pm 99.0$ & $457.8 \pm 142.3$ & $471.6 \pm 73.5$ & $418.1 \pm 141.6$ \\
\hline & \multicolumn{5}{|l|}{ Male } \\
\hline & CTR & SP & SES1 & SES2 & SES3 \\
\hline IL-6 (pg/mg prot) & $1083.5 \pm 476.1$ & $1098.7 \pm 411.3$ & $1061.7 \pm 449.8$ & $873.4 \pm 120.0$ & $1063.7 \pm 280.2$ \\
\hline TNF- $\alpha$ (pg/mg prot) & $764.9 \pm 244.1$ & $634.0 \pm 195.3$ & $883.7 \pm 373.1$ & $837.8 \pm 171.5$ & $742.7 \pm 260.0$ \\
\hline
\end{tabular}

a Data are expressed as mean + SEM.

and water (not shown) consumption and behaviours. Based on these findings, SES was well tolerated at daily doses of 28.5, 57 and $285 \mathrm{mg} / \mathrm{kg} \mathrm{BW} /$ day for 90 days in the rats and there is no evidence for systemic and liver adverse effects and toxicity. Furthermore, there were no signs of oxidative stress or inflammation in the liver, even with the maximum dose of SES used, demonstrating the safety of this dietary supplement.

These findings suggest that silicon-rich spirulina does not induce adverse physiological or biochemical nor pathological changes and could have potential applications in several situations in which oxidative stress and/or inflammation are increased, such as cardiovascular diseases for example. This is in accordance with Yang et al. (2011) and Heussner et al. (2012) relative to spirulina, and corroborates the findings of Buesen et al. (2014) and Horie et al. (2014) although silicon was in the form of silicon dioxide nanoparticles in these two last studies. Elsewhere, further investigations are needed to understand by which mechanisms SES could protect the antioxidant system.

\section{Conflict of interest}

The authors declare that there are no conflicts of interest.

\section{Aknowledgements}

The authors are grateful to Phyco-Biotech company for its interest in this work and its generous gift of the spirulina. Joris Vidé 
was supported by a "CIFRE grant" (Convention Industrielle de Formation par la Recherche, no. 0084/2012) from Phyco-Biotech (Montpellier, France) and the French "Association Nationale de la Recherche et de la Technologie".

\section{References}

Allen, W.M., 1950. A simple method for analyzing complicated absorption curves used in the colorimetric determination of urinary steroids. J. Clin. Endocrinol. 10, 71-83.

Anonymous, 2004. Opinion of the scientific panel on dietetic products, nutrition and allergies on a request from the commission related to the tolerable upper intake level of silicon. EFSA J. 60, 1-11.

Bailly, Y., Duprat, P., 1990. Normal blood cell values. Rat. Hemopoietic Syst. 27-38. doi:10.1007/978-3-642-84110-1; Monographs on Pathology of Laboratory Animals.

Beers, R.F., Sizer, I.W., 1952. A spectrophotometric method for measuring the breakdown of hydrogen peroxide by catalase. J. Biol. Chem. 195, 133-140.

Bond, R., McAuliffe, J.C., 2003. Silicon biotechnology: new opportunities for carbohydrate science. Aust. J. Chem. 56, 7-11.

Borrás, C., Gambini, J., López-Grueso, R., Pallardó, F.V., Viña, J., 2010. Direct antioxidant and protective effect of estradiol on isolated mitochondria. Biochim. Biophys. Acta $1802,205-211$.

Boudard, D., Forest, V., Pourchez, J., Boumandi, N., Tomatis, M., Fubini, B., et al., 2014. In vitro cellular responses to silicon carbide particles manufactured through the Acheson process: impact of physico-chemical features on pro-inflammatory and pro-oxidative effects. Toxicol. In Vitro 28, 856-865.

Buesen, R., Landsiedel, R., Sauer, U.G., Wohlleben, W., Groeters, S., Strauss, V., et al., 2014. Effects of $\mathrm{SiO}_{2}, \mathrm{ZrO}_{2}$, and $\mathrm{BaSO}_{4}$ nanomaterials with or without surface functionalization upon 28-day oral exposure to rats. Arch. Toxicol. 88, 1881-1906.

Calomme, M.R., Vanden Berghe, D.A., 1997. Supplementation of calves with stabilized orthosilicic acid. Effect on the $\mathrm{Si}, \mathrm{Ca}, \mathrm{Mg}$, and $\mathrm{P}$ concentrations in serum and the collagen concentration in skin and cartilage. Biol. Trace Elem. Res. 56, 153-165.

Carlisle, E.M., 1976. In vivo requirement for silicon in articular cartilage and connective tissue formation in the chick. J. Nutr. 106, 478-484.

Chakrabarti, M., Haque, A., Banik, N.L., Nagarkatti, P., Nagarkatti, M., Ray, S.K., 2014. Estrogen receptor agonists for attenuation of neuroinflammation and neurodegeneration. Brain Res. Bull. 109, 22-31.

Chamorro, G.A., Herrera, G., Salazar, M., Salazar, S., Ulloa, V., 1988. Subchronic toxicity study in rats fed Spirulina. J. Pharm. Belg. 43, 29-36.

Deb, U., Lomash, V., Raghuvanshi, S., Pant, S.C., Vijayaraghavan, R., 2012. Effects of 28 days silicon dioxide aerosol exposure on respiratory parameters, blood biochemical variables and lung histopathology in rats. Environ. Toxicol. Pharmacol. 34, 977-984.

Deng, R., Chow, T.J., 2010. Hypolipidemic, antioxidant, and antiinflammatory activities of microalgae Spirulina. Cardiovasc. Ther. 28, e33-e45.

EFSA, 2004. Opinion of the scientific panel on dietetic products, nutrition and allergies on a request from the commission related to the tolerable upper intake level of silicon. EFSA J. 60, 1-11.

Exley, C., 1998. Silicon in life: a bioinorganic solution to bioorganic essentiality. J. Biol. Inorg. Chem. 69, 139-144.

Fallon, M.T., 1996. Rats and mice. In: Laber-Laird, K., Swindle, M.M., Flecknell, P. (Eds.), Handbook of Rodent and Rabbit Medicine. Pergamon/Elsevier Science Ltd., Oxford, pp. 1-38.

Faure, P., Lafond, J.L., 1995. Measurement of plasma sulfhydryl and carbonyl groups as a possible indicator of protein oxidation. In: Favier, A.E., Cadet, J., Kalnyanaraman, B., Fontecave, M., Pierre, J.L. (Eds.), Analysis of Free Radicals in Biological Systems. Birkauser Press, Basel, Boston, Berlin, pp. 237-248.

Flohé, L., Günzler, W.A., 1984. Assays of glutathione peroxidase. Methods Enzymol. $105,114-121$.

Heussner, A.H., Mazija, L., Fastner, J., Dietrich, D.R., 2012. Toxin content and cytotoxicity of algal dietary supplements. Toxicol. Appl. Pharmacol. 265, 263-271.

Horie, M., Nishio, K., Kato, H., Endoh, S., Fujita, K., Nakamura, A., et al., 2014. Evaluation of cellular effects of silicon dioxide nanoparticles. Toxicol. Mech. Methods 24, 196-203.
Ingri, N., 1978. Aqueous silicic acid, silicates and silicate complexes. In: Bendz, G. Lindquist, I. (Eds.), Biochemistry of Silicon and Related Problems. Plenum Press, New York, pp. 3-50.

Jugdaohsingh, R., 2007. Silicon and bone health. J. Nutr. Health Aging 11, 99-110.

Jugdaohsingh, R. Anderson, S.H., Tucker, K.L., Elliott, H., Kiel, D.P., Thompson, R.P. et al., 2002. Dietary silicon intake and absorption. Am. J. Clin. Nutr. 75, 887-893.

Knight, C.T.G., Kinrade, S.D., 2001. A primer on the aqueous chemistry of silicon. In: Datnoff, G.H., Snyder, G.H., Korndöfer, G.H. (Eds.), Silicon in Agriculture. Elsevier Amsterdam, pp. 57-84. 8.

Loeper, J., Goy-Loeper, J., Rozensztajn, L., Fragny, M., 1979. The antiatheromatous action of silicon. Atherosclerosis 33, 397-408.

Loeper, J., Goy, J., Fragny, M., Troniou, R., Bedu, O., 1988. Study of fatty acids in atheroma induced in rabbits by an atherogenic diet with or without silicon I.V. treatment. Life Sci. 42, 2105-2112.

Maehira, F., Motomura, K., Ishimine, N., Miyagi, I., Eguchi, Y., Teruya, S., 2011. Soluble silica and coral sand suppress high blood pressure and improve the related aortic gene expressions in spontaneously hypertensive rats. Nutr. Res. 31, 147-156.

Marklund, S., 1976. Spectrophotometric study of spontaneous disproportionation of superoxide anion radical and sensitive direct assay for superoxide dismutase. J. Biol. Chem. 251, 7504-7507.

Marklund, S., Marklund, G., 1974. Involvement of the superoxide anion radical in the autoxidation of pyrogallol and a convenient assay for superoxide dismutase. Eur. J. Biochem. 47, 469-474.

Mazo, V.K., Gmoshinski, I.V., Zorin, S.N., 2007. New food sources of essential trace elements produced by biotechnology facilities. Biotechnol. J. 2, 1297-1305.

Moraillon, R., Legeay, Y., Boussarie, D., Sénécat, O., 2010. Dictionnaire pratique de thérapeutique chien, chat et NAC. MASSON.

National Research Council, 1985. Guide for the Care and the Use of Laboratory Animals. Publication no. 85-23(rev.). National Institutes of Health, Bethesda, MD.

Reeves, P.G., Nielsen, F.H., Fahey, G.C., Jr., 1993. AIN-93 purified diets for laboratory rodents: final report of the American Institute of Nutrition ad hoc writing committee on the reformulation of the AIN-76 rodent diet. J. Nutr. 123, 19391951.

Refitt, D.M., Jugdaohsingh, R., Thompson, R.P.H., Powell, J.J., 1999. Silicic acid: its gastrointestinal uptake and urinary excretion in man and effects on aluminium excretion. J. Inorg. Biochem. 76, 141-147.

Refitt, D.M., Ogston, N., Jugdaohsingh, R., Cheung, H.F.J., Evans, B.A.J., Thompson, R.P.H., et al., 2003. Orthosilicic acid stimulates collagen type I synthesis and osteoblastic differentiation in human osteoblast-like cells in vitro. Bone 32, 127-135.

Reuter, S., Gupta, S.C., Chaturvedi, M.M., Aggarwal, B.B., 2010. Oxidative stress, inflammation, and cancer: how are they linked? Free Radic. Biol. Med. 49, 1603-1616.

Romain, C., Gaillet, S., Carillon, J., Vidé, J., Ramos, J., Izard, J.C., et al., 2012. Vineatro and cardiovascular disease: beneficial effects of a vine-shoot phenolic extract in a hamster atherosclerosis model. J. Agric. Food Chem. 60, 11029-11036.

Seaborn, C.D., Nielsen, F.H., 1993. Silicon: a nutritional beneficence for bones, brains and blood vessels? Nutr. Today 28, 13-18.

Shwaery, G.T., Vita, J.A., Keaney, J.F., 1998. Antioxidant protection of LDL by physiologic concentrations of estrogens is specific for 17-beta-estradiol. Atherosclerosis 138 255-262.

Skuland, T., Ovrevik, J., Lag, M., Schwarze, P., Refsnes, M., 2014. Silica nanoparticles induce cytokine responses in lung epithelial cells through activation of a p38/TACE/TGF-alpha/EGFR-pathway and NF-kappa B signalling. Toxicol. Appl. Pharmacol. 279, 76-86.

Smith, S.K., Krohn, R.I., Mallia, A.K., Provenzano, M.D., Fujimoto, E.K., Goeke, N.M., et al., 1985. Measurement of protein using bicinchoninic acid. Anal. Biochem. $150,76-85$.

Sunderman, F.W., Jr., Marzouk, A., Hopfer, S.M., Zaharia, O., Reid, M.C., 1985. Increased lipid peroxidation in tissues of nickel chloride-treated rats. Ann. Clin. Lab. Sci. $15,229-236$.

Wu, J., Williams, D., Walter, G.A., Thompson, W.E., Sidell, N., 2014. Estrogen increases Nrf2 activity through activation of the PI3K pathway in MCF-7 breast cancer cells. Exp. Cell Res. 328, 351-360.

Yang Y., Park, Y., Cassada, D.A., Snow, D.D., Rogers, D.G., Lee, J., 2011. In vitro and in vivo safety assessment of edible blue-green algae, Nostoc commune var. sphaeroides Kützing and Spirulina plantensis. Food Chem. Toxicol. 49, 1560-1564 